The operation was performed once for acute inflammatory glaucoma (after a prodromal attack) with a good result; smooth scar with good tension six months after operation; and once for chronic inflammatory glaucoma with a less good result: T. 5.5/2 Schiötz.

Finally, we have performed the operation four times for secondary glaucoma. On one patient the same eye was operated upon twice for staphyloma corneae with transitory effect only. On the other two patients the operations were performed quite recently.

The number of patients is, as shown, too small to make a definite statement regarding the results in these forms of glaucoma. On the whole, however, the efficacy of all fistula-forming operations is more doubtful in cases other than glaucoma simplex.

\title{
ENTOPTIC APPEARANCES OF SENILE CATARACT BY
}

\section{A. G. EAST} TRURO

THESE sketches representing the entoptic images of senile cataract as observed over a period of more than six years were kindly sent to me by one of my patients, and seem to me to be of rather

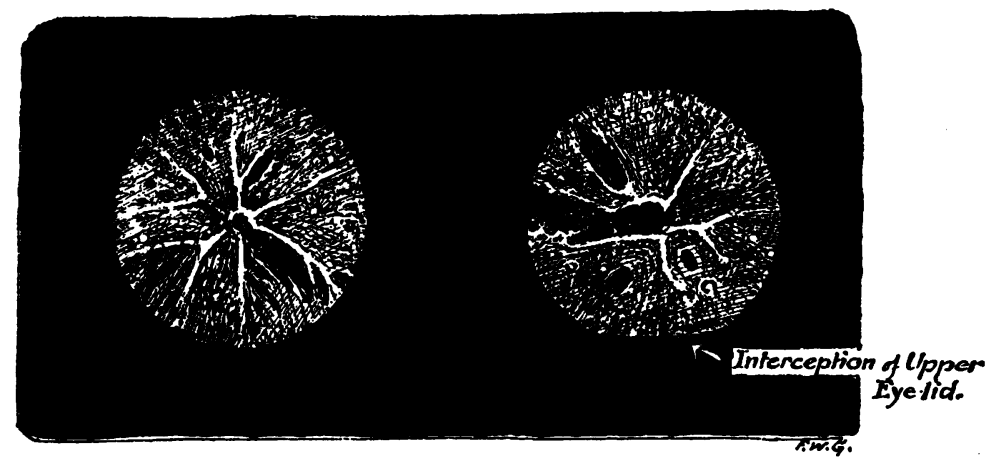

Left Eye. Fig. 1. Right Eye.

Images in Reverse of Crystalline Lenses.

unusual interest, as it is not often that one comes across people with the ability to represent accurately, in the manner shown, the pathological changes occurring in their own lenses.

The sketches were made with the aid of a pin-hole in a black card, held between the window and the eye. 


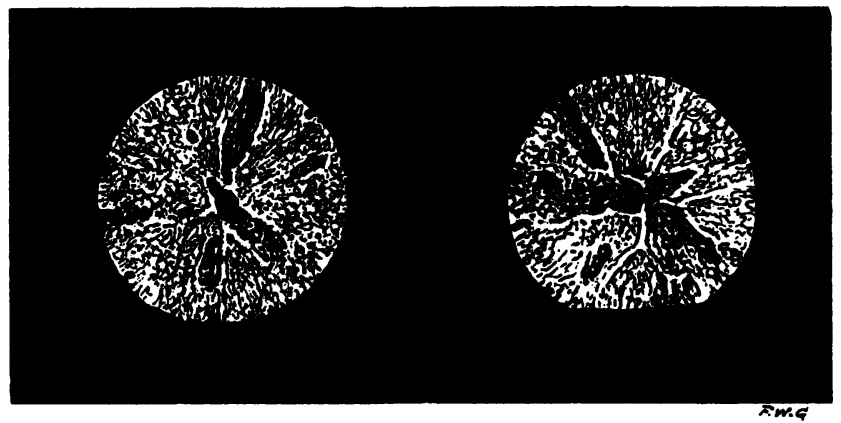

Fig. 2.

LEFT EYE. Right Eye.

Inverted Image Crystalline Lenses. Sketched January 25, 1919. See also No. 3.

No. 1, drawn in October, 1915, represents a stage of the process in which the vision was:-

Right $6 / 9$ ? with +0.75 cyl. Ax: $90^{\circ}=6 / 6$.

Left $6 / 6$ ? ? with $-0.25 \mathrm{sph} .=6 / 6$.

When Nos. 2 and 3 were'sketched there was no record made of his visual acuity.

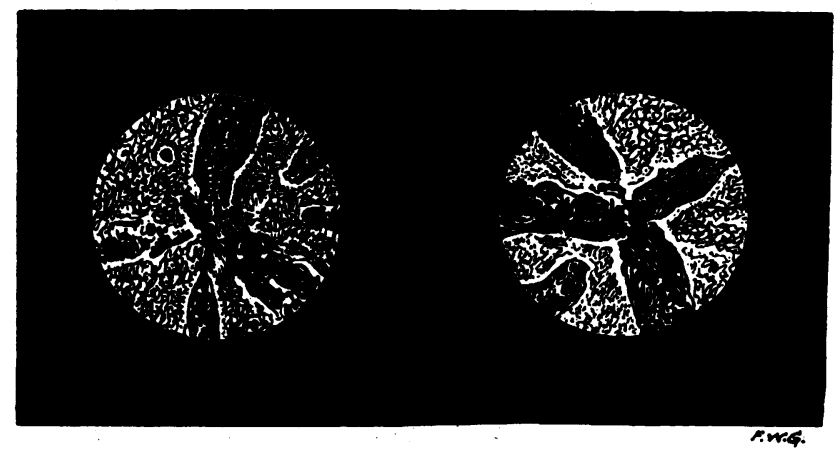

FIG. 3.

LEFT EYE.

RIGHT Eye.

Inverted Image Crystalline Lenses. Sketched January 30, 1922.

No. 3 represents the condition as it was when I saw him in January, 1922 .

His vision with correction was-

R. 6/9. L. 6/12.

The changes in his lenses are clearly seen with the plane mirror of an ophthalmoscope. 